\title{
Atributos do solo e dimensão fractal em ambientes naturais e transformados na região amazônica
}

A região Amazônica apresenta ambientes naturais e ambientes transformados, sendo a pecuária a principal atividade. A utilização inadequada do solo tem ocasionado a degradação de suas propriedades físicas, químicas e biológicas. O objetivo deste trabalho foi realizar um levantamento bibliográfico sobre as relações entre os atributos físicos e carbono orgânico do solo com a sua qualidade e a possibilidade de utilizar a dimensão fractal como instrumento para avaliar a textura do solo, em ambientes naturais e transformados na Amazônia. Os atributos físicos e a matéria orgânica do solo podem ser diretamente afetados pelos diferentes sistemas de uso e manejo. Diante disso, verificou-se que atributos do solo, como a estabilidade de agregados, porosidade, densidade, umidade, resistência do solo à penetração, textura e matéria orgânica, podem ser utilizados como indicadores de qualidade do solo. Verificou-se, também, diante dos resultados revistos, que a dimensão fractal pode servir como instrumento na avaliação da textura do solo, uma vez que a textura é uma composição fracionada de partículas de solo.

Palavras-chave: Ambientes; Atributos do Solo; Dimensão Fractal.

\section{Soil attributes and fractal dimension in natural and transformed environments in the amazon region}

\begin{abstract}
The Amazon region presents natural environments and transformed environments, with livestock being the main activity. Inadequate soil use has resulted in the degradation of its physical, chemical and biological properties. The objective of this work was to perform a bibliographical survey about the relationships between physical attributes and organic carbon of the soil with its quality and the possibility of using the fractal dimension as an instrument to evaluate soil texture in natural and transformed environments in the Amazon. The physical attributes and organic matter of the soil can be directly affected by different systems of use and management. Thus, soil attributes, such as aggregate stability, porosity, density, moisture, soil penetration resistance, texture and organic matter, can be used as indicators of soil quality. It has also been verified from the results that the fractal dimension can serve as an instrument in the evaluation of soil texture, since the texture is a fractional composition of soil particles.
\end{abstract}

Keywords: Environments; Soil Atributes; Fractal Dimension.

José Cezar Frozzi

Universidade Federal do Amazonas, Brasil http://lattes.cnpq.br/2757266093020607 http://orcid.org/0000-0001-6528-2035 icfrozzi@gmail.com

\section{Anderson Cristian Bergamin (iD}

Universidade Federal de Rondônia, Brasil http://lattes.cnpq.br/3961477986363222 http://orcid.org/0000-0002-3159-2219 andersonbergamin@hotmail.com

José Maurício da Cunha

Universidade Federal do Amazonas, Brasil http://lattes.cnpq.br/3425545536495518 maujmc@gmail.com
Milton César Costa Campos

Universidade Federal do Amazonas, Brasil http://lattes.cnpq.br/9041514924498589 mcesarsolos@gmail.com

Alan Ferreira Leite de Lima

Universidade Federal do Amazonas, Brasil http://lattes.cnpq.br/2890744656048373 icfrozzi@gmail.com

Wildson Benedito Mendes Brito

Universidade Federal do Amazonas, Brasil http://lattes.cnpq.br/3605269722545071 wild.brito@gmail.com

\section{Igor Hister Lourenço (iD) \\ Universidade Federal do Amazonas, Brasi http://lattes.cnpq.br/0894326537418462 http://orcid.org/0000-0001-5282-7649 icfrozzi@gmail.com}

Wellington Luiz de Melo Silva

Universidade Federal do Amazonas, Brasil http://lattes.cnpq.br/1885298831563447 well.luiz.83@gmail.com

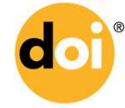

DOI: 10.6008/CBPC2179-6858.2018.002.0020

\section{Referencing this:}

FROZZI, J. C.; BERGAMIN, A. C.; CUNHA, J. M.; CAMPOS, M. C. C.; LIMA, A. F. L.; BRITO, W. B. M.; LOURENÇO, I. H.; SILVA, W. L. M.. Atributos do solo e dimensão fractal em ambientes naturais e transformados na região amazônica. Revista Ibero Americana de Ciências Ambientais, v.9, n.2, p.231-243, 2018. DOI: http://doi.org/10.6008/CBPC21796858.2018 .002 .0020 


\section{INTRODUÇÃO}

A região Amazônica está situada na parte norte da América do Sul, com cerca de 6 milhões de km² (VALE JÚNIOR et al., 2011), possuindo a maior floresta tropical do mundo, sendo o maior reservatório de diversidade biológica do planeta e onde ainda persistem ambientes naturais. Até meados da década de 60, a sua exploração era de forma extrativista, sem impactos ambientais significativos, entretanto, posteriormente, a exploração se intensificou para integrar a região ao processo produtivo e econômico do país.

A pecuária, uma das atividades econômicas que mais se desenvolveram na ocupação e na utilização das terras, contribuiu para que grandes extensões de florestas fossem desmatadas e cedessem lugar às pastagens cultivadas (COSTA, 2000). O desmatamento na Amazônia brasileira tem como principais causas diretas a pecuária, a agricultura de larga escala e a agricultura de corte e queima. Dessas causas, a expansão da pecuária bovina é a mais importante, provocando, muitas vezes, impactos ambientais negativos e até mesmo irreversíveis (RIVERO et al., 2009).

Segundo Sá et al. (2010), a degradação dos atributos físicos, químicos e biológicos do solo pode ser provocada por sua utilização inadequada. Com isso, torna-se necessário a avaliação desses atributos para que sejam propostas técnicas que visam minimizar e/ou inibir essa degradação. Pelo contexto, o objetivo deste trabalho foi realizar um levantamento bibliográfico sobre as relações entre os atributos físicos e carbono orgânico do solo com a sua qualidade e a possibilidade de utilizar a dimensão fractal como ferramenta para avaliar a textura do solo, em ambientes naturais e transformados na Amazônia.

\section{METODOLOGIA}

Para a realização do presente trabalho foram necessários levantamentos de literaturas pertinentes aos assuntos: atributos do solo e teoria dos fractais. Esses levantamentos foram realizados em livros, publicações em periódicos conceituados, banco de teses e dissertações e sites de órgãos reguladores, servindo de base para o atendimento dos objetivos propostos.

\section{DISCUSSÃO TEÓRICA}

\section{Usos e manejos do solo em ambientes amazônicos}

A região Amazônica apresenta ambientes naturais e ambientes transformados, sendo a pecuária a principal atividade (RIVERO et al., 2009). O solo é o recurso natural essencial para o desenvolvimento de atividades, de onde o ser humano obtém seus alimentos, além de ser responsável pela manutenção da qualidade ambiental e, consequentemente, pela sanidade de plantas, animais e seres humanos (SHARMA et al., 2005). Os ambientes naturais apresentam integração direta entre a cobertura vegetal e os atributos físicos, químicos e biológicos do solo, decorrentes de processos essenciais relacionados aos ciclos biogeoquímicos, acumulação e decomposição da matéria orgânica de resíduos vegetais (serapilheira e raízes) e microclima (KARA et al., 2008). 
Quando conservado em estado natural, com vegetação nativa, o solo apresenta características físicas adequadas ao bom desenvolvimento das plantas, pois é constituído de elementos que tendem para um estado de equilíbrio, sendo que, a alteração em qualquer um desses elementos pode repercutir em todo o conjunto. Nessas condições, o volume de solo explorado pelas raízes é relativamente grande. No entanto, o uso e manejo inadequado do solo tem ocasionado a degradação dos atributos físicos, químicos e biológicos (SÁ et al., 2010), causando, muitas vezes, impactos ambientais negativos, como exemplo a sua desestruturação, compactação, redução da fertilidade, perda da matéria orgânica e diminuição da diversidade e quantidade de organismos (LEITE et al., 2010).

Com o desmatamento, o uso e manejo inadequado do solo as propriedades físicas sofrem alterações, geralmente desfavoráveis ao desenvolvimento das plantas, pois causam o rompimento do equilíbrio do ecossistema e cuja intensidade é função da natureza, atributos dos solos, condições litológicas estruturais, clima e relevo (NOVAES FILHO et al., 2007; CAMARGO et al., 2010). O manejo inadequado do solo, juntamente com as limitações naturais tais como a umidade excessiva e alta temperatura, alto grau de acidez e baixo suprimento de nutrientes do solo na maior parte da região, têm sido as causas da produtividade limitada de alimentos e fibras na Amazônia brasileira (LUIZÃO et al., 2008).

As diversas operações de manejo como o tráfego intenso de máquinas agrícolas ou pisoteio de animais, perdas de matéria orgânica ou mesmo fenômenos da pedogênese, associadas às precipitações pluviométricas intensas que são características dessa região, alteram a estrutura do solo, podendo causar efeitos negativos como a compactação e a erosão do solo (PETEAN, 2011; KRAMER, 2012; MONCADA et al., 2014b). Os sistemas de cultivo ao modificarem os atributos físicos do solo podem prejudicar sua qualidade (NIERO, 2009).

Práticas sustentáveis no uso dos recursos naturais, em especial do solo e da água, destacaram-se como tema relevante, devido, principalmente, ao aumento de atividades antrópicas (ARAÚJO et al., 2010). Para garantir um período duradouro de cultivo, a avaliação deste recurso é de extrema necessidade, pois a conservação e a manutenção dos solos tornam-se indispensáveis para a garantia de seu potencial produtivo (GALETI, 1973), garantido também, dessa maneira, sua sustentabilidade. Essa avaliação é complexa e deve ser realizada em função de um conjunto de indicadores específicos, denominados atributos e suas interrelações, já que se tem verificado que indicadores isolados não são suficientes para explicar a perda ou o ganho potencial dos cultivos de determinado solo (SANTOS, 2010).

\section{Atributos físicos do solo em ambientes amazônicos}

Os atributos físicos, químicos e biológicos dos solos são importantes componentes de sua produtividade, visto que as plantas necessitam de solos bem estruturados, sendo por isso a seleção e a utilização adequada de cada tipo de solo de fundamental importância para a manutenção da qualidade e da produtividade do sistema (PIGNATARO NETTO et al., 2009; BOGNOLA et al., 2010).

A transformação de ambientes naturais em sistemas de produção, como a agropecuária que ocorre na Amazônia, provoca alterações significativas nos atributos físicos do solo. Devido ao revolvimento do solo, 
tráfego contínuo de máquinas e equipamentos agrícolas, há alteração estrutural, aumento da densidade do solo, resistência do solo à penetração e redução da macroporosidade, causando a degradação cumulativa da qualidade física do solo ao longo dos anos de cultivo (CARVALHO et al., 2008; COLLARES et al., 2008; ROQUE et al., 2010).

A utilização inadequada do solo, sobretudo por meio da adoção de sistemas convencionais, tem ocasionado a degradação de suas propriedades físicas, químicas e biológicas (SÁ et al., 2010). Como exemplo, a desestruturação e compactação, perdas de solo e nutrientes por erosão e lixiviação, redução da fertilidade, oxidação acelerada da matéria orgânica, perdas de carbono $(C)$, nitrogênio $(N)$, fósforo $(P)$ e dos reservatórios de nutrientes associados a matéria orgânica e a diminuição da quantidade e diversidade de organismos do solo (LEITE et al., 2010).

$\mathrm{Na}$ área de pastagem o pisoteio dos animais pode afetar os atributos físicos do solo pela deformação de sua estrutura, promover mudanças na densidade e porosidade, influir na resistência mecânica à penetração e até provocar a compactação do solo. Por outro lado, o conteúdo orgânico pode melhorar a estabilidade dos agregados e ter relevância nos atributos químicos dos solos tropicais (NETO et al., 2009). Sistemas de manejo intensivo, que causam degradação no carbono orgânico, reduzem a estabilidade dos agregados, vindo a ocorrer compactação, erosão e consequentemente queda de produtividade. A formação de camadas compactadas reduz a atividade biológica e a macroporosidade no perfil do solo, aumentando a densidade, o que proporciona maior resistência física à expansão radicular (JIMENEZ et al., 2008).

Como visto, o desenvolvimento das plantas depende da qualidade do solo que está diretamente relacionada aos seus atributos físicos, que podem ser afetados pelos diversos sistemas de uso e manejo. 0 êxito de projetos agrícolas depende, muitas vezes, destes atributos e a presença e desenvolvimento de espécies vegetais estão diretamente relacionados a estas propriedades, assim como o movimento de água no solo para o deslocamento de seus nutrientes, poluentes químicos dissolvidos e partículas (FREITAS et al., 2013).

O estudo do comportamento dos atributos físicos do solo é de suma importância na escolha do sistema de manejo a ser empregado nesse solo e, ao longo do tempo, permite quantificar a magnitude e a duração das alterações provocadas por diferentes sistemas de manejo (REICHERT et al., 2009), por serem parâmetros sensíveis, esses atributos são importantes para estabelecer se houve diminuição ou melhoria da qualidade do solo, em referência a um determinado sistema de manejo.

Estudos mais específicos evidenciaram que os atributos físicos do solo variam de um local para outro, apresentando continuidade ou dependência espacial, dependendo do manejo adotado e material de origem dos solos (CAMARGO et al., 2008; BOTTEGA et al., 2011). Souza et al. (2009), encontraram dependência espacial para os atributos físicos na cultura de cana-de-açúcar, no entanto, Campos et al. (2012) verificaram que ocorre variabilidade espacial na resistência do solo à penetração e no conteúdo de água do solo, em área sob cultivo de mandioca. 


\section{Estabilidade de agregados}

A estrutura do solo pode ser considerada uma das características mais importante do solo. Basicamente, é definida como o ordenamento das partículas primárias juntamente com componentes minerais e orgânicos, formando as unidades estruturais do solo, os 'agregados' (CAPECHE, 2008), tendo influência na movimentação de água, transferência de calor, aeração, densidade e porosidade. Porém, a estrutura por si só não é considerada um fator de crescimento das plantas, mas influencia em quase todos os fatores de crescimento vegetal, como fornecimento de água e fluxo de nutrientes, dentre outros (AMARO FILHO et al., 2008; MUELLER et al., 2013) e, ao contrário da textura, é dinâmica, alterando-se constantemente pela ação do homem e da natureza.

A estrutura do solo pode ser avaliada tanto em campo como em laboratório. Existem vários métodos para essa avaliação e novas metodologias podem surgir (FERREIRA, 2010). Dentre os métodos de avaliação da estrutura do solo existe uma classificação em diretos e indiretos. Nos métodos indiretos estão aqueles cuja avaliação é realizada a partir de propriedades do solo, como condutividade hidráulica, taxa de infiltração de água, densidade e distribuição e tamanho dos poros; nos métodos diretos estão os que avaliam as características estruturais morfológicas, como microscopia, análise de imagens, medição de estabilidade dos agregados em laboratório e avaliação visual da estrutura a campo (MONCADA et al., 2014a). Para que o solo tenha sustentabilidade em diferentes sistemas de uso e manejo é de fundamental necessidade a utilização de métodos que avaliam a sua qualidade e estabilidade estrutural (ARATANI et al., 2009).

\section{Porosidade do solo}

A composição básica do solo é dada por partículas sólidas, sendo que na organização destas partículas encontram-se espaços de diferentes tamanhos, denominados poros, que fazem parte da fração volumétrica do solo preenchida pelo ar e pela água (RIBEIRO et al., 2007). A porosidade é um importante atributo do solo e confere condições propícias ao desenvolvimento radicular e a existências de organismos vivos. Conforme Lepsch (2011), cerca da metade do volume do solo corresponde aos poros e eles são divididos em dois tipos: os macroporos $(>0,06 \mathrm{~mm})$, onde a água e o ar encontram maior facilidade de movimentação; e os microporos $(<0,06 \mathrm{~mm})$, que devido à capilaridade, a água permanece por mais tempo, sendo estes, de fundamental importância para o desenvolvimento das plantas, principalmente em períodos de déficit hídrico (SANCHEZ, 2012).

De acordo com Reinert et al. (2008), os manejos do solo que utilizam plantas de cobertura com sistemas radiculares abundantes e vigorosos, além de reciclarem nutrientes em seu perfil, adicionam matéria orgânica e melhora a estabilidade dos agregados, conferindo ao solo maior porosidade e resistência mecânica sendo a qualidade da porosidade diretamente relacionada a esta resistência mecânica. A porosidade em um solo sob manejo convencional, apresenta maior sensibilidade à compressão unidimensional aplicada, devido ao menor espaço poroso, quando comparado com o solo sob plantio direto (BORTOLUZZI et al., 2008). Em sistemas onde não ocorre o revolvimento do solo, constata-se uma lenta 
diminuição da porosidade, em plantio direto estudos têm observado uma diminuição no volume total de poros, de macroporosidade e um aumento na microporosidade (FERNANDES et al., 2007).

\section{Densidade do solo}

A densidade do solo é um instrumento importante na avaliação de impactos ambientais sobre o solo, sendo definida como o peso seco de um determinado volume de solo, pois leva em consideração a porosidade do solo. Reflete o arranjo das partículas definindo as características do sistema poroso. A permeabilidade do solo, por sua vez, é inversamente proporcional à densidade do solo, sendo importante para indicar a capacidade de armazenamento de água para as plantas e para que se possam encontrar as melhores práticas de conservação do solo e água (AZEVEDO et al., 2004).

A densidade do solo representa sua situação estrutural, tendo relação direta com a umidade e a resistência do solo à penetração. Assim, em condições de baixa qualidade do solo, as raízes das plantas têm seu crescimento limitado (BLAINSKI et al., 2008). A densidade é um atributo que mede características distintas ao solo. Ao comparar diferentes classes texturais, uma densidade de $1,5 \mathrm{~g} . \mathrm{cm}^{-3}$, em solo arenoso não apresenta problema de compactação, já em solo argiloso com este mesmo índice apresentaria uma forte compactação (SANCHEZ, 2012). Na avaliação de qualidade do solo é uma propriedade física considerada como indicador, pois está relacionada com a sua estrutura, porosidade e taxa de infiltração de água, levando em consideração a textura deste solo (ALVES et al., 2007).

Em sistemas de cultivo que não efetuam o preparo do solo observa-se um aumento na disponibilidade de água para as plantas, mesmo com um aumento da densidade, quando comparado com sistema de cultivo convencional, porém podem ocorrer variações, dependendo do teor de matéria orgânica e da textura do solo (CAVALIERI et al., 2006).

\section{Umidade do solo}

Na agricultura, a umidade do solo constitui uma das mais importantes propriedades, relacionandose diretamente entre solo-planta e a vida microbiológica. A umidade do solo influencia na aeração, temperatura e na sua resistência mecânica, que são afetadas pela densidade e distribuição do tamanho do espaço poroso. Esses atributos físicos se inter-relacionam e regulam o desenvolvimento radicular, com reflexo na produtividade das plantas cultivadas (COLLARES et al., 2006).

Um fenômeno que demonstra as boas condições físicas de um solo é a infiltração de água, pois está relacionada às suas condições estruturais, influenciadas pelos diferentes sistemas de uso e manejo, pois estes interferem na quantidade e dimensão do espaço poroso, sendo considerada como indicador de qualidade (ALVES et al., 2007). Esses diferentes sistemas de uso e manejo do solo interferem diretamente na capacidade de retenção e evaporação de água. Gava (2010), em experimento com diferentes taxas de resíduos de palha de trigo, em Latossolo Vermelho eutroférrico, observou a evaporação diminui com o aumento da cobertura de palha, intensificando este efeito com precipitações frequentes. Atributos como condutividade hidráulica, 
a tensão da água, a difusão de gases e a resistência do solo à penetração, estão diretamente relacionados com a quantidade de água neste solo (CAVALIERI et al., 2006).

\section{Resistência do solo à penetração}

A resistência do solo à penetração é utilizada há vários anos, com aplicações em diversos campos da pesquisa agronômica. É um atributo importante para a caracterização e manejo do solo, sendo relacionada ao teor de umidade e densidade do solo. Segundo Reichert et al. (2010), a resistência do solo à penetração tem sido utilizada como indicador de camadas compactadas e mudanças nas propriedades físicas do solo associadas aos seus horizontes. Uma resistência do solo à penetração entre 1,0 - 3,5 MPa pode limitar o crescimento radicular (BEUTLER et al., 2004; BLAINSKI et al., 2008).

A compactação é um dos mais sérios danos causados ao solo devido à exploração excessiva do mesmo e pode ser definida como sendo a ação mecânica imposta ao solo, provocando uma redução em seus espaços porosos, sendo a relação entre o volume de vazios e o volume de sólidos. Os solos agrícolas podem ser compactados pelo pisoteio de animais em pastejo, pelas raízes de árvores, sendo os efeitos mais significativos os provindos da mecanização agrícola (SOARES FILHO, 1992). Nessa lista, incluem-se também as características físicas do solo, o teor de água e a presença de resíduos culturais, que são fatores importantes no processo de compactação. As propriedades físicas e mecânicas dos solos influenciam negativamente ou positivamente em sua compactação e, desta forma, na trafegabilidade e desenvolvimento radicular (DANIEL et al., 1995). Portanto, a resistência do solo à penetração é um dos atributos físicos do solo que influencia o crescimento de raízes servindo como um bom indicador na avaliação de sua qualidade física.

\section{Textura do solo}

A textura do solo representa à proporção relativa em que se encontram os diferentes tamanhos de partículas, em determinada massa de solo. Refere-se, especificamente, as partículas ou frações de areia, silte e argila e é uma das características físicas mais estáveis do solo (SANCHEZ, 2012). É o atributo físico do solo que menos sofre alteração ao longo do tempo pela influência do manejo. Durante a classificação do solo em um determinado local, a textura dos diferentes horizontes é a mais importante propriedade a ser determinada. Partindo desta informação muitas conclusões importantes podem ser tomadas, como a definição da distribuição do diâmetro dos poros do solo por meio do teor de argila (FREITAS et al., 2013).

A textura possui influência no comportamento do solo, quando submetido a pressões externas, pois determina o atrito entre as partículas e o tipo de ligação entre elas. Em geral, quanto maiores as partículas do solo, menor sua compressibilidade e agregação (MACEDO et al., 2010). Suzuki et al. (2014), ao avaliarem a textura do solo em um Argissolo Vermelho distrófico sob pastagem cultivada, floresta nativa e povoamento de eucalipto no Rio Grande do Sul, observaram incremento no teor de argila e cascalho e redução do teor de areia, de acordo com aumento da profundidade. A variação da textura nas diferentes áreas pode estar associada à variabilidade natural, associada ao relevo, ou até mesmo aos possíveis processos erosivos ocorridos ao longo dos anos. 


\section{Matéria orgânica do solo em ambientes amazônicos}

A matéria orgânica desempenha uma função muito importante sobre as características físicas, químicas e biológicas do solo, sendo um indicador de qualidade nos diferentes sistemas de uso e manejo (NASCIMENTO et al., 2009). Auxilia na recuperação da porosidade, em função de ser um condicionador biofísico e sua baixa densidade, em comparação com os minerais, reduz a densidade do solo, sendo que

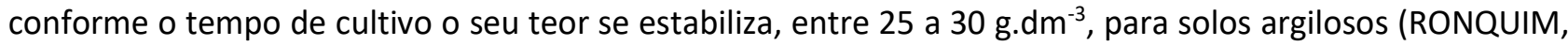
2010).

Segundo Ribas (2010), a importância do material orgânico em solos tropicais e subtropicais altamente intemperizados é tanto no fornecimento de nutrientes às culturas, retenção de cátions e complexação de elementos tóxicos e micronutrientes, como na estabilidade da estrutura, aeração, infiltração e retenção de água. Tem como principais componentes o carbono (C) e o nitrogênio (N), com estoques variando de acordo com as adições (resíduos orgânicos) e perdas (erosão, oxidação por microrganismos), sendo que em solos naturais é influenciada por fatores edafoclimáticos enquanto que em solos cultivados sofre com o sistema de manejo empregado (SOUZA et al., 2009).

Uso inapropriado do solo pode rapidamente levar a uma degradação da matéria orgânica e, consequentemente, alterações nas propriedades físicas, químicas e biológicas do solo (JAKELAITIS et al., 2008). A inclusão de sistemas de uso e manejo que visam à conservação do solo através da adição e manutenção de matéria orgânica é fundamental para uma boa estrutura. Essa prática serve como fonte de energia para os microrganismos e atuam como agentes de estabilização dos agregados (CAMPOS et al., 1995).

Hickmann et al. (2012), verificaram, em um experimento de 23 anos constituído de quatro sistemas de manejo, que o sistema plantio direto na camada de 0,0-0,5 cm aumentou o teor carbono orgânico total e o estoque de carbono orgânico total, comparado com o manejo convencional. Porém as camadas 0,5-10; 1020 e 20-40 cm, não demonstraram variações entre os manejos, mas um decréscimo em profundidade, demonstrando, assim, o efeito dos diferentes manejos no aporte de carbono orgânico do solo.

\section{Dimensão Fractal}

A geometria fractal foi introduzida nos anos 70 pelo matemático polonês Benoit Mandelbrot. De modo contrário à geometria euclidiana, a fractal se apresenta como ferramenta eficientemente capaz de quantificar e qualificar séries de dados temporais e espaciais descrevendo com maior precisão a sua complexidade (HOTT et al., 2005). Na tentativa de definir as variações de certas formas e fenômenos matemáticos, chegou à conclusão de que as variações pequenas e grandes têm uma conexão, uma familiaridade, e obedecem a uma constante: a escala, na qual em função de ampliações chega-se a perceber variações de tamanho, entretanto, nem sempre de forma. Desse modo, o matemático polonês criou o termo fractal, com a necessidade de definir um conceito baseado nas frações. 
A dimensão fractal possibilita melhorar a capacidade de representar estruturas complexas e irregulares da natureza, para toda estrutura que em partes dela se assemelham ao todo, ou seja, existem partes autossimilares, estatisticamente dentro da estrutura global. Isso sugere a presença do fenômeno de escala e de um nível de tendência, o qual pode ser medido através da dimensão fractal, que é uma medida de complexidade (MANDELBROT, 1982; RUSS, 1995). Portanto, essa técnica visa a caracterização de fenômenos naturais, irregulares, com maior precisão que as análises convencionais, permitindo uma análise com menores distorções da realidade.

Os modelos fractais têm sido aplicados para a caracterização da distribuição do tamanho de partículas no solo, expresso em termos de sua massa (CASTRIGNANÒ et al., 1999; TYLER et al., 1992; PERFECT et al., 1995), facilmente obtida pela Equação (1).

$$
\frac{M(r<R)}{M_{T}}=\left(\frac{R}{R_{L}}\right)^{3-D}
$$

Em que $M$ é a massa acumulada das partículas de tamanho $r$ menores do que $R$ determinado pelo diâmetro das peneiras, $M_{T}$ é a massa total, $R_{L}$ é o parâmetro que estima o tamanho da maior partícula e $D$ é a dimensão fractal da massa. Essa equação é limitada pela variação de $D$ com $0<D<3$ e é aplicada para descrever a distribuição do tamanho da partícula do solo seco. Os dois parâmetros do modelo, a dimensão fractal $D$ e o tamanho da maior partícula $R_{L}$, são definidas como ferramentas descritivas para a textura do solo.

As estimativas não viciadas de $D$ e $R_{L}$, para cada ponto amostral, são obtidas por regressão não-linear, através do método de Gauss-Newton para o cálculo de estimadores de mínimos quadrados (SOUZA, 1998; SAS INSTITUTE, 1985; PERFECT et al., 1992). As representações das estimativas da dimensão fractal (D) obtidas pela transformação log-log da massa acumulada $\frac{M(r<R)}{M_{T}}$ e diâmetro da partícula $\left(\frac{R}{R_{L}}\right)$, podem ser definidas pela Equação (1). O cálculo da dimensão fractal tem sido usado para descrever qualitativamente a fragmentação das partículas do solo (PROSPERINI et al., 2008; LIU et al., 2009; PARENT et al., 2011; XU et al., 2013; CUNHA et al., 2016). Altos valores para a dimensão fractal, em diferentes usos do solo, foram indicados para avaliar melhor a qualidade do solo (XU et al., 2013).

Os resultados do trabalho de Cunha et al. (2016), com a fragmentação dos agregados, realizado na região Sul do Amazonas, corroboram com a afirmação anterior, onde a dimensão fractal da distribuição do tamanho de partículas no solo teve maior valor médio em sistema de uso de agrofloresta $(D F=1,971)$, evidenciando solos de melhor qualidade em comparação com as demais áreas de cana-de-açúcar $(D F=1,758)$, mandioca $(D F=1,796)$, floresta nativa $(D F=1,453)$ e pastagem $(D F=1,401)$, com menores valores da dimensão fractal.

Os resultados de Cunha et al. (2016), foram para agregados do solo, os quais possuem em seus interiores espaços porosos, que influenciam para que os valores de dimensão fractal sejam menores quando comparados com os valores da dimensão fractal da textura dos solos, por exemplo. Prova disso, são os valores encontrados por Xiao et al. (2014), na região montanhosa de Yimeng, na China, onde a dimensão 
fractal da distribuição do tamanho das partículas (textura) do solo variou entre 2,141 e 2,526 e era sensível ao processo de 'engrossamento' do solo que ocorreu nas áreas perturbadas.

Da mesma forma, Xu et al. (2013), avaliando a dimensão fractal da distribuição de tamanho de partícula (textura) do solo em diferentes tipos de vegetação e com tipos de solos semelhantes, em uma bacia hidrográfica no meio do Vale do Dan, na China, verificaram que os distúrbios antropogênicos tiveram um grande impacto nas dimensões fractais de diferentes tipos de uso do solo na área de estudo, apresentando valores de dimensão fractal variando entre 2,59 e 2,96. Os resultados dos dois últimos estudos demonstram que a análise da dimensão fractal da distribuição de tamanho de partícula (textura) do solo oferece uma abordagem útil para quantificar e avaliar o grau de degradação do solo entre tipos de solo semelhantes.

No estudo de Xiao et al. (2014), no Planalto de Loess, na China, onde estudaram o comportamento da dimensão fractal em dez padrões típicos de uso do solo, obtendo resultados que mostraram que a dimensão fractal da textura do solo foi influenciada significativamente pelas profundidades do solo, padrões de uso do solo e suas interações, com valores entre 2,600 e 2,821.

Para Xiao et al. (2014), os resultados demostraram que a dimensão fractal foi sensível aos diferentes perfis de intemperismo, com valores entre 2,597 e 2,921 . Os resultados deste estudo demonstram que a dimensão fractal pode ser usada para caracterização da distribuição do tamanho das partículas (textura) do solo e ser considerada como um indicador informativo para refletir o grau de intemperismo de diferentes perfis do solo em ravinas na região montanhosa granítica, no Sul da China. Conforme Cunha et al. (2016), esses resultados podem mostrar que uma única função do tipo lei de potência é necessária para descrever a fragmentação dos agregados do solo. Este fator pode caracterizar todas as análises das amostras do solo, com inclinações das retas diferentes para cada ponto amostral.

\section{CONCLUSÕES}

Os atributos físicos e a matéria orgânica do solo podem ser diretamente afetados pelos diferentes sistemas de uso e manejo. Diante disso, verificou-se que atributos do solo, como a estabilidade de agregados, porosidade, densidade, umidade, resistência do solo à penetração, textura e matéria orgânica, podem ser utilizados como indicadores de qualidade do solo. Verificou-se, também, diante dos resultados revistos, que a dimensão fractal pode servir como instrumento na avaliação da textura do solo, uma vez que a textura é uma composição fracionada de partículas de solo.

\section{REFERÊNCIAS}

ALVES, M. C.; SUZUKI, L. G. A. S.; SUZUKI, L. E. A. S.. Densidade do solo e infiltração de água como indicadores da qualidade física de um Latossolo Vermelho distrófico em recuperação. Revista Brasileira de Ciência do Solo, v.31, n.4, p.617-625, 2007. DOI: http://dx.doi.org/10.1590/S010006832007000400002

AMARO FILHO, J.; ASSIS JÚNIOR, R. N.; MOTA, J. C. A.. Física do solo: conceitos e aplicações. Fortaleza: Imprensa Universitária, 2008.
ARATANI, R. G.; FREDDI, O. S.; CENTURION, J. F.; ANDRIOLI, I.. Qualidade física de um Latossolo Vermelho acriférrico sob diferentes sistemas de uso e manejo. Revista Brasileira de Ciência do Solo, v.33 n.3, 2009. DOI: http://dx.doi.org/10.1590/S0100-06832009000300020

ARAÚJO, F. S.; SALVIANO, A. A. C.; LEITE, L. F. C.; SOUZA, Z. M.; SOUSA, A. C. M.. Physical quality of a yellow Latossol under integrated crop-livestock system. Revista Brasileira de Ciência do Solo, Viçosa, v.34, n.3, p.717-723, 2010. DOI: http://dx.doi.org/10.1590/S0100-06832010000300013 
AZEVEDO, A. C.; DALMOLIN, R. S. D.. Solos e Ambiente: uma introdução. Santa Maria: Pallotti, 2004.

BLAINSKI, É.; TORMENA, C. A.; FIDALSKI, J.; GUIMARÃES R. M. L.. Quantificação da degradação física do solo por meio da curva de resistência do solo à penetração. Revista Brasileira de Ciência do Solo, v.32, n.3, p.975-983, 2008. DOI: http://dx.doi.org/10.1590/S0100-06832008000300007

BEUTLER, A. N.; CENTURION, J. F.; SILVA, A. P.; ROQUE, C. G.; FERRAZ, M. V.. Compactação do solo e intervalo hídrico ótimo na produtividade do arroz sequeiro. Pesquisa Agropecuária Brasileira, v.39, n.6, p.575-580, 2004. DOI: http://dx.doi.org/10.1590/S0100-204X2004000600009

BOGNOLA, I. A.; DEDECEK, R. A.; LAVORANTI, O. J.; HIGA, A. R.. Influência de propriedades físicohídricas do solo no crescimento de Pinus taeda. Pesquisa Florestal Brasileira, Colombo, v.30, n.61, p.37-49, 2010.

BORTOLUZZI, E. C.; SILVA, V. R.; PETRY, C.; CECCHETTI, D. Porosidade e retenção de água em um Argissolo sob manejos convencional e direto submetido a compressões unidimensionais. Revista Brasileira de Ciência do Solo, v.32 n.4, 2008. DOI: http://dx.doi.org/10.1590/S010006832008000400009

BOTTEGA, E. L.; BOTTEGA, S. P.; SILVA, S. A.; QUEIROZ, D. M. SOUZA, C. M. A.; RAFULL, L. Z. L.. Variabilidade espacial da resistência do solo à penetração em um Latossolo Vermelho distroférrico. Revista Brasileira de Ciências Agrárias, v.6, n.2, p.331-336, 2011. DOI: http://10.5039/agraria.v6i2a882

CAMARGO, L. A.; MARQUES JÚNIOR, J.; PEREIRA, G. T.; HORVAT, R. A.. Variabilidade espacial de atributos mineralógicos de um Latossolo sob diferentes formas do relevo. II - Correlação espacial entre mineralogia e agregados. Revista Brasileira de Ciência do Solo, v.32, n.6, p.2279-2288, 2008. DOI: http://dx.doi.org/10.1590/S0100$\underline{06832008000600007}$

CAMARGO, L. A.; MARQUES JÚNIOR, J.; PEREIRA, G. T.. Spatial variability of physical attributes of an alfisol under diferente hillslope curvatures. Revista Brasileira de Ciência do Solo, v.34, n.3, p.617-630, 2010. DOI: http://dx.doi.org/10.1590/S0100-06832010000300003

CAMPOS, B. C.; REINERT, D. J.; NICOLODI, R.; RUEDELL, J.; PETRERE, C.. Estabilidade estrutural de um Latossolo Vermelho-Escuro distrófico após sete anos de rotação de culturas e sistemas de manejo de solo. Revista Brasileira de Ciência do Solo, v.19, n.1, p.121-126, 1995.

CAMPOS, M. C. C.; RIBEIRO, M. R.; SOUZA JÚNIOR, V. S.; RIBEIRO FILHO, M. R.; ALMEIDA, M. C.. Topossequência de solos na transição campos naturais-floresta na região de Humaitá, Amazonas. Acta Amazônica, Manaus, v.42, n.3, p.387-398, 2012. DOI: http://dx.doi.org/10.1590/S0044$\underline{59672012000300011}$

CAPECHE, C. L.. Noções sobre tipos de estrutura do solo e sua importância para o manejo conservacionista. Comunicado Técnico 51. Rio de Janeiro: Embrapa Solos, 2008.

CARVALHO, L. A.; NETO, V. J. M.; SILVA, L. F.; PEREIRA, J. G.; NUNES, W. A. G. A.; CHAVES, C. H. C.. Resistência mecânica do solo à penetração (RMP) sob cultivo de cana-de-açúcar, no município de Rio Brilhante/MS. Agrarian, v.1, n.2, p.7-22, 2008.

CASTRIGNANÒ, A.; STELLUTI, M.. Fractal geometry and geostatistics for describing the field variability of soil aggregation. Journal of Agricultural Engineering Research., v.73, n.1, p.13-18, 1999. DOI:

https://doi.org/10.1006/jaer.1998.0385

CAVALIERI, K. M. V.; TORMENA, C. A.; VIDIGAL FILHO, P. S.; GONÇALVES, A. C. A.; COSTA, A. C. S.. Efeitos de sistemas de preparo nas propriedades físicas de um Latossolon Vermelho distrófico. Revista Brasileira de Ciência do Solo, v.30, n.1 p.137-147, 2006. DOI: http://dx.doi.org/10.1590/S010006832006000100014

COLLARES, G. L.; REINERT, D. J.; REICHERT, J. M.; KAISER, D. R.. Qualidade física do solo na produtividade da cultura do feijoeiro num Argissolo. Pesquisa Agropecuária Brasileira, v.41, n.11, p.1663-1674, 2006. DOI: http://dx.doi.org/10.1590/S0100-204X2006001100013

COLLARES, G. L.; REINERT, D. J.; REICHERT, J. M.; KAISER, D. R.. Compactação de um Latossolo induzida pelo tráfego de máquinas e sua relação com o crescimento e produtividade de feijão e trigo. Revista Brasileira de Ciências do Solo, v.32, n.3, p.933-942, 2008. DOI: http://dx.doi.org/10.1590/S0100$\underline{06832008000300003}$

COSTA, N. A.. Controle de plantas invasoras em pastagens cultivadas. In: COSTA, N. A.. Pastagens cultivadas na Amazônia. Belém: Embrapa Amazônia Oriental, 2000. p.7298.

CUNHA, J. M.; CAMPOS, M. C. C.; GAIO, D. C.; NOGUEIRA, J. S.; SOARES, M. D. R.; SILVA, D. M. P.; OLIVEIRA, I. A.. Fractal analysis in the description of soil particle-size distribution under different land-use patterns in Southern Amazonas State, Brazil. African Journal of Agricultural Research, v.11 p.23, p.2032-2042, 2016. DOI: http://10.5897/AJAR2015.10649

DANIEL, L. A.; LUCARELLI, J. R.; CARVALHO, J. F.. Efeito do método de preparo do solo na formação e localização de camadas compactadas. In: CONGRESSO BRASILEIRO DE ENGENHARIA AGRÍCOLA, 24. Anais. Viçosa: SBEA/UFV, 1995. p.370.

FERNANDES, F. C. S.; ALVES, M. C.; SILVA, M. M..

Produtividade de culturas e atributos físicos de um Latossolo afetados pelo sistema de manejo. Revista Brasileira de Milho e Sorgo, v.6, n.3, p.297-308, 2007. DOI: http://dx.doi.org/10.18512/1980-6477/rbms.v6n3p297-308

FERREIRA, M. M.. Caracterização Física do Solo: Física do Solo. Viçosa: Sociedade Brasileira de Ciência do Solo, 2010.

FREITAS, L.; CASAGRANDE, J. C.; OLIVEIRA, I. A.; MORETI, T. C. C. F.; CARMO, D. A. B.. Avaliação de Atributos Químicos e Físicos de Solos com Diferentes Texturas Cultivados com Cana-de-Açúcar. Enciclopédia Biosfera, Goiânia, v.9, n.17, p.362-374, 2013.

GALETI, P. A.. Conservação do Solo. 2 ed. Campinas: Instituto Campineiro de Ensino Agrícola, 1973.

GAVA, R.. Evaporação da água do solo sob diferentes taxas de cobertura com resíduos vegetais. Dissertação (Mestrado em Agronomia) - Universidade Estadual de Maringá, Maringá, 2010

HICKMANN, C.; COSTA, L. M.. Estoque de carbono no solo e agregados em Argissolo sob diferentes manejos de longa duração. Revista Brasileira de Engenharia Agrícola e Ambiental, v.16, n.10, 2012. DOI: http://dx.doi.org/10.1590/S1415-43662012001000004

JAKELAITIS, A.; SILVA, A. A.; SANTOS, J. B.; VIVIAN, R. Qualidade da camada superficial de solo sob mata, pastagem e áreas cultivadas. Pesquisa Agropecuária Tropical, Goiânia, v.38, n.2, p.118-127, 2008. 
JIMENEZ, R. L.; GONÇALVES, W. G.; ARAÚJO FILHO, J. V.; ASSIS, R. L.; PIRES, F. R.; SILVA, G. P.. Crescimento de plantas de cobertura sob diferentes níveis de compactação em um Latossolo Vermelho. Revista Brasileira de Engenharia Agrícola e Ambiental, Campina Grande, v.12, n.2, p.116121, 2008. DOI: http://dx.doi.org/10.1590/S141543662008000200002

KARA, Ö.; BOLAT, I.. Soil microbial biomass $\mathrm{C}$ and $\mathrm{N}$ changes in relation to forest conversion in the northwestern Turkey. Land Degradation \& Development, Chichester, v.19, n.4, p.421-428, 2008. DOI: http://10.1002/ldr.850

KRAMER, L. F. M.. Atributos químicos e físicos de um Latossolo sob plantio direto em zonas de manejo com diferentes produtividades. Dissertação (Mestrado em Agronomia) - Universidade Estadual do Centro-Oeste, Guarapuava, 2012.

LEITE, L. F.; GALVÃO, S. R.; HOLANDA NETO, M.; ARAÚJO, F. S.; IWATA, B. F.. Atributos químicos e estoques de carbono em Latossolo sob plantio direto no cerrado do Piauí. Revista Brasileira de Engenharia Agrícola e Ambiental, Campina Grande, v.14, n.12, p.1273-1280, 2010. DOI: http://dx.doi.org/10.1590/S1415-43662010001200004

LEPSCH, I. F.. 19 lições de pedologia. São Paulo: Oficina de Textos, 2011.

LIU, X.; ZHANG, G.; HEATHMAN, G. C.; WANG, Y.; HUANG, C.. Fractal features of soil particlesize distribution as affected by plant communities in the forested region of Mountain Yimeng, China. Geoderma, v.154, n.10, p.123-130, 2009. DOI: https://doi.org/10.1016/j.geoderma.2009.10.005

LUIZÃO, F. J.; FEARNSIDE, P. M.; CERRI, C. E. P.; LEHMANN, J.. The maintenance of soil fertility in Amazonian managed systems. p. 311-336. In: KELLER, M.; BUSTAMANTE, M.; GASH, J.; SILVA DIAS, P.. Amazonia and Global Change. Geophysical Monograph Series. 186 ed. Washington: American Geophysical Union (AGU), 2008.

MACEDO, V. R. M.; SILVA A. J. N.; CABEDA M. S. V.. Influência de tensões compressivas na pressão de pré-compactação e no índice de compressão do solo. Revista Brasileira de Engenharia Agrícola e Ambiental, v.14, n.8, p.856-862, 2010. DOI: http://dx.doi.org/10.1590/S141543662010000800010

MANDELBROT, B. B.. The fractal geometry of nature. New York: W. H. Freeman, 1982.

MONCADA, M. P.; GABRIELS, D.; LOBO, D.; REY, J. C.; CORNELIS, W. M.. Visual field assessment of soil structural quality in tropical soils. Soil \& Tillage Research, v.139, n.1, p.8-18, 2014a. DOI:

https://doi.org/10.1016/j.still.2014.01.002

MONCADA, M. P.; PENNING, L. H.; TIMMD, L. C.; GABRIELS D.; CORNELIS, W. M.. Visual examinations and soil physical and hydraulic properties for assessing soil structural quality of soils with contrasting textures and land uses. Soil \& Tillage Research, v.140, n.2, p.20-28, 2014b. DOI: https://doi.org/10.1016/j.still.2014.02.009

MUELLER, L.; SHEPHERD, G.; SCHINDLER, U.; BALL, B. C.; MUNKHOLM, L. J.; HENNINGS, V.; SMOLENTSEVA, E.; RUKHOVIC, O.; LUKIN, S.; HUI, C.. Evaluation of soil structure in the framework of an overall soil quality rating. Soil \& Tillage Research, v.127, n.3, p.74-84, 2013. DOI: https://doi.org/10.1016/j.still.2012.03.002

NASCIMENTO, P. C.; BAYER, C.; SILVA NETTO, L. F.; VIAN, A. C.; VIEIRO, F.; MACEDO, V. R. M.; MARCOLIN, É.. Sistemas de manejo e a matéria orgânica de solo de várzea com cultivo de arroz. Revista Brasileira de Ciência do Solo, v.33, n.6, p.1821-1827, 2009. DOI: http://dx.doi.org/10.1590/S0100$\underline{06832009000600030}$

NETO, M. S.; PICCOLO, M. C.; SCOPEL, E.; JÚNIOR, C. C.; CERRI, C. C.; BERNOUX, M.. Carbono total e atributos químicos com diferentes usos do solo no cerrado. Acta Scientiarum Agronomy, Maringá, v.31, n.4, p.709-717, 2009. DOI: http://dx.doi.org/10.4025/actasciagron.v31i4.792

NIERO, L. A. C.. Avaliações Visuais como Índice de Qualidade do Solo e sua Validação por Análises Físicas e Químicas em um Latossolo Vermelho Distroférico com Usos e Manejos Distintos. Dissertação (Mestrado em Agronomia) - Instituto Agronômico, Campinas, 2009.

NOVAES FILHO, J. P.; COUTO, E. G.; OLIVEIRA, V. A.; JOHNSON, M. S.; LEHMANN, J.; RIHA, S. S.. Variabilidade especial de atributos físicos de solo usada na identificação de classes pedológicas de microbacias na Amazônia meridional. Revista Brasileira de Ciência do Solo, v.31, n.1, p.91-100, 2007. DOI: http://dx.doi.org/10.1590/S0100$\underline{06832007000100010}$

PARENT, L. E.; PARENT, S.; KÄTTERER, T.; EGOZCUE, J. J. Fractal and compositional analysis of soil aggregation. In: INTERNATIONAL WORKSHOP ON COMPOSITIONAL DATA ANALYSIS, 4. Anais. 2011.

PERFECT, E.; RASIAH, V.; KAY, B. D.. Fractal dimension of soil aggregatesize distributions calculated by number and mass. Soil Science Society American Journal, v.56, n.5, p.14071409, 1992. DOI: http://10.2136/sssaj1992.03615995005600050012x

PERFECT, E.; KAY, B. D.. Brittle fracture of fractal cubic aggregates. Soil Science Society American Journal, v.59, n.4, p.969-974, 1995. DOI:

http://10.2136/sssaj1995.03615995005900040002x

PETEAN, L. P.. Qualidade física de um Latossolo Vermelho distroférrico sob integração lavoura-pecuária. Tese (Doutorado em Agronomia) - Universidade Estadual de Maringá, Maringá, 2011.

PIGNATARO NETTO, I. T.; KATO, E; GOEDERT, W. J.. Atributos físicos e químicos de um latossolo Vermelho-amarelo sob pastagens com diferentes históricos de uso. Revista Brasileira de Ciência do Solo, Viçosa, v.3, n.5, p.1441-1448, 2009. DOI: http://dx.doi.org/10.1590/S010006832009000500034

PROSPERINI, N.; PERUGINI, D.. Particle size distributions of some soils from the Umbria Region (Italy): Fractal analysis and numerical modeling. Geoderma, v.145, n.3, p.185-195, 2008. DOI: https://doi.org/10.1016/j.geoderma.2008.03.004

REICHERT, J. M.; KAISER, D. R.; REINERT, D. J.; RIQUELME, U. F. B.. Variação temporal de propriedades físicas do solo e crescimento radicular de feijoeiro em quatro sistemas de ambiente. Pesquisa Agropecuária Brasileira, v.44, n.3, p.310-319. 2009. DOI: http://10.1590/S0100$\underline{204 \times 2009000300013}$

REICHERT, J. M.; REINERT, D. J.; SUZUKI, L. E. A. S.; HORN, R. Mecânica do Solo. In: VAN LIER, Q. J.. Física do Solo. Viçosa: Sociedade Brasileira de Ciência do Solo, 2010. p.29-102.

REINERT, D. J.; ALBUQUERQUE, J. A.; REICHERT, J. M.; AITA, C.; ANDRADA, M. M. C.. Limites Críticos de Densidade do Solo para o Crescimento de Raízes de Plantas de Cobertura em Argissolo Vermelho. Revista Brasileira de Ciência do Solo, v.32, n.5, p.1805-1816, 2008. DOI: http://dx.doi.org/10.1590/S0100-06832008000500002 
RIBEIRO, K. D.; MENEZES, S. M.; MESQUITA, M. da G. B. de F.; SAMPAIO F. M. T.. Propriedades físicas do solo, influenciadas pela distribuição de poros, de seis classes de solos da região de Lavras-MG. Ciência Agrotécnica, v.31, n.4, p.1167-1175, 2007. DOI: http://dx.doi.org/10.1590/S141370542007000400033

RIBAS, C.. Caracterização da fertilidade atual dos solos da região de Guarapuava - PR. Dissertação (Mestrado em Agronomia) - Universidade Estadual do Centro-Oeste, Guarapuava, 2010.

RIVERO, S.; ALMEIDA, O.; ÁVILA, S.; OLIVEIRA, W.. Pecuária e desmatamento: uma análise das principais causas diretas do desmatamento na Amazônia. Nova Economia, Belo Horizonte, v.19, n.1, p.41-66, 2009. DOI: http://dx.doi.org/10.1590/S0103-63512009000100003

ROQUE, A. A. O.; SOUZA, Z. M.; BARBOSA, R. S.; SOUZA, G. S.. Controle de tráfego agrícola e atributos físicos do solo em área cultivada com cana-de-açúcar. Pesquisa Agropecuária Brasileira, v.45, n.7, p.744-750, 2010. DOI: http://dx.doi.org/10.1590/S0100-204X2010000700016

RONQUIM, C. C.. Conceitos de fertilidade do solo e manejo adequado para as regiões tropicais. Boletim de Pesquisa e Desenvolvimento. Campinas: Embrapa Monitoramento por Satélite, 2010.

SÁ, I, B.; CUNHA, T. J.F.; TEIXEIRA, A. H. C.; ANGELOTTI, F.; DRUMOND, F. M.. Desertificação no Semiárido brasileiro. ICID+18. In: CONFERÊNCIA INTERNACIONAL: CLIMA SUSTENTABILIDADE E DESENVOLVIMENTO EM REGIÕES SEMIÁRIDAS, 2. Anais. Fortaleza, 2010.

SANCHEZ, E.. Propriedades físicas do solo e produtividade de soja em sucessão a plantas de cobertura de inverno. Dissertação (Mestrado em Produção Vegetal) - Universidade Estadual do Centro-Oeste, Guarapuava, 2012.

SANTOS, V. B.. Atributos de solos sob cultivo de frutíferas em sistemas de manejo convencional, em transição e orgânico no norte do estado do Piauí. Tese (Doutorado em Produção Vegetal) - Universidade Estadual Paulista, Jaboticabal, 2010.

SAS INSTITUTE. SAS user's guide: statistics. 5 ed. Cary, 1985
SHARMA, K. L.; MANDAL, U.K.; SRINIVAS, K.; VITTAL, K. P. R.; MANDAL, B.; GRACE, J. K.; RAMESH, V.. Longterm soil management effects on crop yelds and soil quality in dryland Alfisol. Soil Till. Res., v.83, n.8, p.246-259, 2005. DOI: http://10.1016/j.still.2004.08.002

SOARES FILHO, R.. Identificação e avaliação dos sistemas motomecanizados de preparo periódico do solo, usados no município de Rio Verde/GO. Dissertação (Mestrado em Engenharia Agrícola) - Universidade Federal de Viçosa, Viçosa, 1992.

SOUZA, G. S.. Introdução aos modelos de regressão linear e não-linear. Brasília: Embrapa-SPI/Embrapa-SEA, 1998.

SOUZA, Z. M.; MARQUES JÚNIOR, J.; PEREIRA, G. T.. Geoestatística e atributos do solo em áreas cultivadas com cana-de-açúcar. Ciência Rural, v.40, n.5, p.48-56, 2009. DOI: http://dx.doi.org/10.1590/S0103-84782009005000243

SUZUKI, L. E. A. S.; LIMA, C. L. R.; REINERT, D. J.; REICHERT, J. M.; PILLON, C. M.. Estrutura e armazenamento de água em um Argissolo sob pastagem cultivada, floresta nativa e povoamento de eucalipto no Rio Grande do Sul. Revista Brasileira de Ciência do Solo, Viçosa, v.38, n.1, p.94-106, 2014. DOI: http://dx.doi.org/10.1590/S010006832014000100009

TYLER, S. W.; WHEATCRAFT, S. W.. Fractal scaling of soil particle size distributions: analysis and limitations. Soil Science Society American Journal, v.56, n.2, p.362-369, 1992. DOI: http://10.2136/sssaj1992.03615995005600020005x

VALE JÚNIOR, J. F.; SOUZA, M. I. L.; NASCIMENTO, P. P. R. R.; CRUZ, D. L. S.. Solos da Amazônia: etnopedologia e desenvolvimento sustentável. Revista Agro@mbiente,v.5, n.2, p.158-165, 2011. DOI: http://dx.doi.org/10.18227/19828470ragro.v5i2.562

XIAO, L.; XUE, S.; LIU, G. B.; ZHANG, C.. Fractal features of soil profiles under different land use patterns on the Loess Plateau, China. Journal of Arid Land., v.6, n.14, p.550-560. 2014. DOI: http://10.1007/s40333-014-0023-7

XU, G.; LI, Z.; LI, P.. Fractal features of soil particle-size distribution and total soil nitrogen distribution in a typical watershed in the source area of the middle Dan River, China. Catena, v.101, n.9, p.17-23, 2013. DOI: https://doi.org/10.1016/j.catena.2012.09.013

A CBPC - Companhia Brasileira de Produção Científica (CNPJ: 11.221.422/0001-03) detém os direitos materiais desta publicação. Os direitos referem-se à publicação do trabalho em qualquer parte do mundo, incluindo os direitos às renovações, expansões e disseminações da contribuição, bem como outros direitos subsidiários. Todos os trabalhos publicados eletronicamente poderão posteriormente ser publicados em coletâneas impressas sob coordenação da Sustenere Publishing, da Companhia Brasileira de Produção Científica e seus parceiros autorizados. Os (as) autores (as) preservam os direitos autorais, mas não têm permissão para a publicação da contribuição em outro meio, impresso ou digital, em português ou em tradução. 